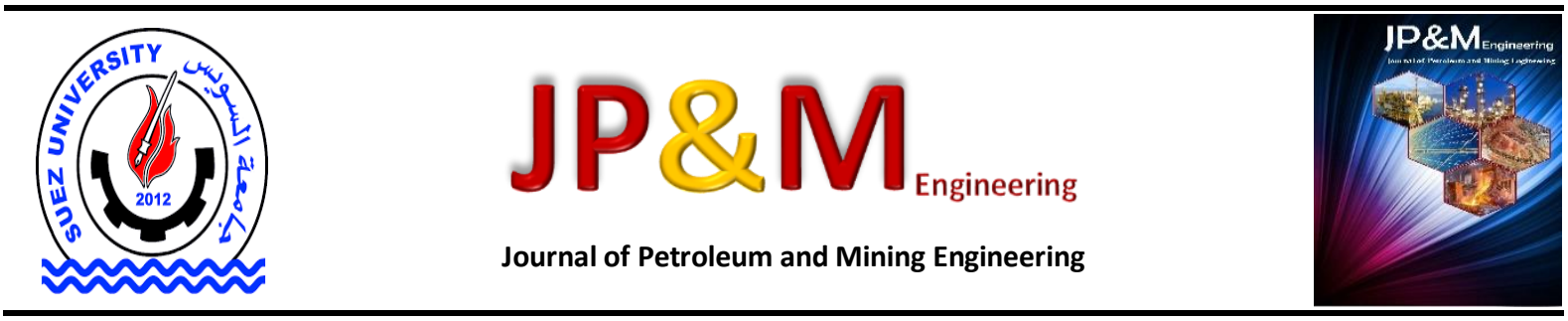

\title{
Drill bits optimization using offset wells analysis and ROP modelling
}

\author{
Yasin, A., ${ }^{a *}$ Farahat, M. S., ${ }^{b}$ Shalaby, S. E. ${ }^{b}$ and Salem, A. M. ${ }^{b}$ \\ aDrilling and Workover Department, Belayiem Petroleum Company, Cairo, Egypt \\ bPetroleum Engineering Department, Faculty of Petroleum and Mining Engineering, Suez University, Egypt \\ *Corresponding Author E-mail: ahmedyasin90@yahoo.com
}

\section{Article Info}

Received: 9 Nov. 2020

Revised: 30 Mar. 2021

Accepted: 30 Apr. 2021

\section{Keywords}

ROP model; multiple regression; analysis; Drill bit optimization

\begin{abstract}
Drill Bits optimization is the key factor to improve the overall drilling efficiency and therefore, reduce the cost and the non-productive time. For this, analysis of the offset field data, modelling before drilling and optimization the drilling parameters are highly required to enhance the drilling efficiency. In this paper, $8 \frac{1}{2}$ " hole of three drilled wells is analysed for the rate of penetration (ROP), drilling cost per meter and the drilling duration of each drill bit. The analysis has shown that the variation in drilling efficiency was due to some factor such as, the type and features of the used drill bit, the bottom hole assembly type and the drilling parameters. ROP model (multiple regression equation) is developed by using a reference well's drilling parameters. The drilling parameters that have been used to develop the ROP model are weight on bit (WOB), revolution per minute (RPM) of the drill string, the drilling torque (TRQ), jet impact force (JIF) of the drill bit and the unconfined compressive strength (UCS) of the formation. Parametric sensitivity study has been carried out using this model to interpret the drilling parameters that have the greatest impact on ROP. Finally, drill bits' with the highest performance are recommended to be used based on analysis of the offset wells and ROP model "multiple regression equation" has been approached to predict the ROP values before drilling to be used in optimization of drill bits' performance in the forthcoming drilled wells.
\end{abstract}

\section{Introduction}

The objective of the drilling operation is to safely execute the drilling plan at optimum performance with the minimum drilling cost and time. The operators are continuously looking for techniques that maximize operation efficiency and minimize the time to drill each section ${ }^{(1 ; 2)}$.

Optimizing the drilling efficiency could only be achieved through three distinct stages; planning and modeling before drilling, monitoring during drilling and analysis the drill bits performance after drilling ${ }^{(3)}$.

Drilling in deeper and harsher conditions well requires more advanced drilling technology and equipment. Therefore, the efficiency of drill bits is increased by improving their quality and this will allow a further increase in the rate of penetration (4).

The challenges in drilling $81 / 2$ " hole section are a lot; the high unconfined compressive strength of conglomerate formation, the torque fluctuation, the high vibrations that were being generated when PDC bits had been used and the high bit wear in a short time. In addition to the previously mentioned challenges, the operator was suffering from the high cost per every drilled meter and the time consumed in drilling that section.

The target in Sidri area is the conglomerate formation. The thickness of Conglomerate ranges from $900 \mathrm{~m}$ to $1500 \mathrm{~m}$ and with the same lithology. Conglomerate formation has high-unconfined compressive strength (UCS) that varies between 15,000 - 35,000 psi. Conglomerate formation is composed mainly of $95 \%$ granitic fragment, which had been formed from basement erosion.

One thousand meter of $81 / 2$ " hole was drilled using more than 7 drill bits. Most drilled wells required more than 20 days to drill $81 / 2$ " section. In the three studied wells, the drilling performance was high. The three wells have been analysed and ROP model has been developed.

The objective of this paper is to present analysis about the drill bits' performance of three drilled 
wells in Sinai Oil Field, establish ROP prediction mathematical model by using a reference well (well with the highest drill bit performance), then test the accuracy of the ROP model by applying the model on the nearby wells and use the ROP model to predict the most effective drilling parameters on the drilling performance.

The developed ROP model is multiple regression, which characterizes an observation factor by several variables, taking into consideration changes of several properties simultaneously. In this paper, the observation factor $(\mathrm{Y})$ is the rate of penetration. Relevant drilling factors make up the regression variables (X1-5) ${ }^{(5)}$. The ROP model built a relationship between the rate of penetration (ROP) and WOB, RPM, TRQ, JIF and UCS.

\section{Methodology}

\section{Analysis of drill bits' performance}

The performance of a drill bits could be analysed using ROP values, the length of drilled interval and the cost per meter per every drill bit.

Equation (1) is applied on every drill bit run and by comparing the cost per meter values among all the drill bits, it would be easily to define the drill bits that achieved high performance and those achieved poor performance.

$\mathrm{C}=\frac{\mathrm{B}+(\mathrm{T}+\mathrm{t}) * \mathrm{R}}{\mathrm{L}}$

C: cost per meter $(\$ / m)$

R: Rig cost per hour $(\$ / h r)$

B: refers to bit cost (\$)

$\mathrm{T}$ : Trip time (hr)

$\mathrm{L}$ : refers to the drilled interval $(\mathrm{m})$

$\mathrm{t}$ : rotating time on bottom $(\mathrm{hr})$

\section{ROP Modelling}

Multiple regression workflow has been carried out on $81 / 2$ " hole section of the reference well (well- $X$ ), the parameters used in multiple regression analysis are in the form of WOB, RPM, torque, JIF and the UCS of the drilled formation, together with the observation factor ROP.

The modified jet impact force given in Eq. (2) was therefore chosen to replace the Flow rate and the standpipe pressure (SPP) in the model ${ }^{(6)}$.

$\mathrm{JIF}=\frac{\mathrm{Qx}(\sqrt{\rho \mathrm{xPbit}}}{58}$

JIF: Jet impact force (psi)

Q: flow rate (GPM)

$\rho:$ drilling fluid density (PPG)

Pbit: pressure drop across the drill bit

The unconfined compressive strength of formation was included in the equation due to the great impact of the formation strength on the drill bits performance, UCS can be calculated using interval transit time $(\Delta t)$ recorded by wire line sonic $\log { }^{(7)}$, given in Eq. (3).

$\mathrm{UCS}(\mathrm{MPA})=\frac{\left(\frac{7682}{\Delta \mathrm{t}}\right)^{2}}{145}$

UCS: unconfined compressive strength, $\mathrm{MPa}$

$\Delta \mathrm{t}: \quad$ interval transit time

The $(Y)$ range represents the ROP, while $(X)$ range is the drilling variables. The depth is considered only as a reference and is not involved in the analysis. The coefficients are then provided by the analysis. The intercept value is represented by the initial value of coefficients (b0). The other coefficients (b1, b2, b3, b4 $\& b 5)$ are then multiplied according to their order with the multiple regression equation's variables $(X 1, X 2$, $X 3, X 4 \& X 5)$. The multiple regression equation that represents the ROP model could be formed as Eq. (4):

$\mathrm{Y}=\mathrm{b} 0+\mathrm{b} 1 \mathrm{X} 1+\mathrm{b} 2 \mathrm{X} 2+\mathrm{b} 3 \mathrm{X} 3+\mathrm{b} 4 \mathrm{X} 4+$ b5X5

This equation could be written in terms of ROP and drilling parameters as:

Modelled ROP $=\mathrm{b} 0+\mathrm{b} 1 \mathrm{WOB}+\mathrm{b} 2 \mathrm{RPM}+$ b3 Torque + b4 JIF + b5 UCS

\section{Implementation of the multiple regression workflow in Microsoft Excel software}

The multiple regression procedure shown in Figure (1) is applied on the reference well (Well-X). In Microsoft Excel, multiple regression data analysis is carried out by entering Input-Y-range from column (J) which represents the actual ROP values and $X$-range from columns (E, $F, G, H$, and $I)$, the multiple regression workflow has been carried out providing the multiple regression coefficients listed in cells Y2O to Y25, shown in Figure (2) "yellow colored".

Equation (5) is implemented for row no.4 of drilling data. The equation calculates the modelled ROP for each row by changing the row reference number, shown in Figure (3).

$\mathrm{K}=(\$ \mathrm{Y} \$ 20)+(\$ \mathrm{Y} \$ 21 * \mathrm{E} 5)+(\$ \mathrm{Y} \$ 22 * \mathrm{~F} 5)+$

$(\$ Y \$ 23 * \mathrm{G} 5)+(\$ Y \$ 24 * \mathrm{H} 5)+(\$ Y \$ 25 * \mathrm{I} 5)$

Figure 1 Multiple Regression Procedures flowchart 


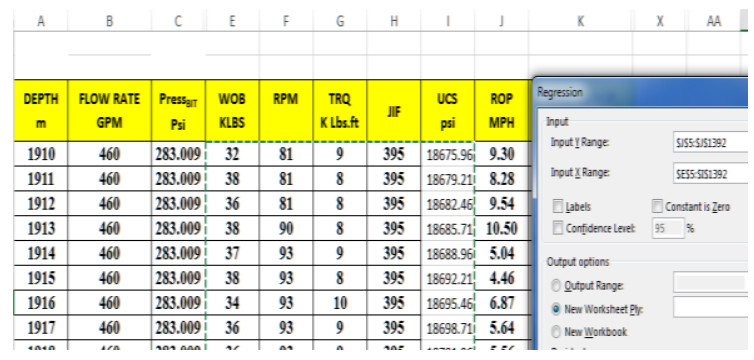

Figure $\mathbf{2}$ multiple regression data analysis (Microsoft Excel)

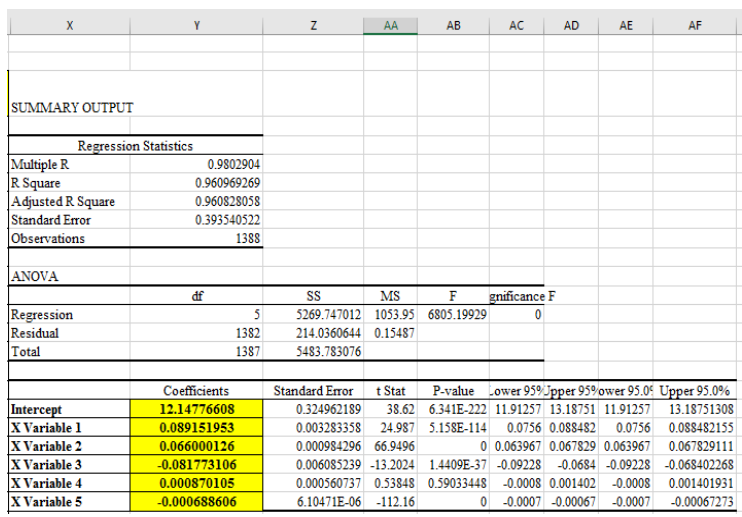

Figure 3 multiple regression equation is applied in Microsoft Excel

\section{Testing the developed ROP model on two nearby wells}

After applying Eq. (6) on the reference well (Well$X)$, the multiple regression equation is tested on two nearby wells (Well-Y \& Well-Z) with the same drilling criteria; the bottom hole assemblies and drill bits and similar geological structure. Using the coefficients resulting from multiple regression workflow and with applying the equation on the two wells, predicted ROP values have been computed.

The results (ROP profile) of implementing the multiple regression equation on the two wells are compared with the actual ROP profile to test the reliability of the multiple regression equation. If the predicted ROP profile is similar and close in values to the actual one, the ROP model proves that it could be applicable on that area.

\section{ROP model and parametric sensitivity}

A parametric sensitivity analysis is carried out in this study to find out which drilling parameters have a greater impact on the ROP. The drilling parameters that were used in the ROP model (WOB, RPM, torque, JIF \& UCS) are increased by $10 \%$ one by one. The new predicted ROP values are compared with the original predicted ROP. By this way, the most effective drilling parameters could be determined, and the optimum drilling parameters can be applied in drilling the next wells. The final step in the parametric sensitivity is to apply the most effective drilling parameters with the increase percentage on the ROP model. The predicted ROP values have been the highest.

\section{Results and discussions}

\section{Analysis of the three wells}

The three studied wells have been analyzed using bit run cost equation for each drill bit. In addition, ROP values and the length of the drilled interval per each drill bit are also taken into consideration in the analysis study, shown in table 1.

It's clearly obvious the hybrid bit (KM524) had achieved the highest performance in the upper part of $81 / 2 "$ hole and the sting blade PDC bit had achieved good performance in the lower part of $81 / 2$ " hole where the compressive strength is higher.

Table 1 analysis of Drill bits performance in wells (X, Y \& Z)

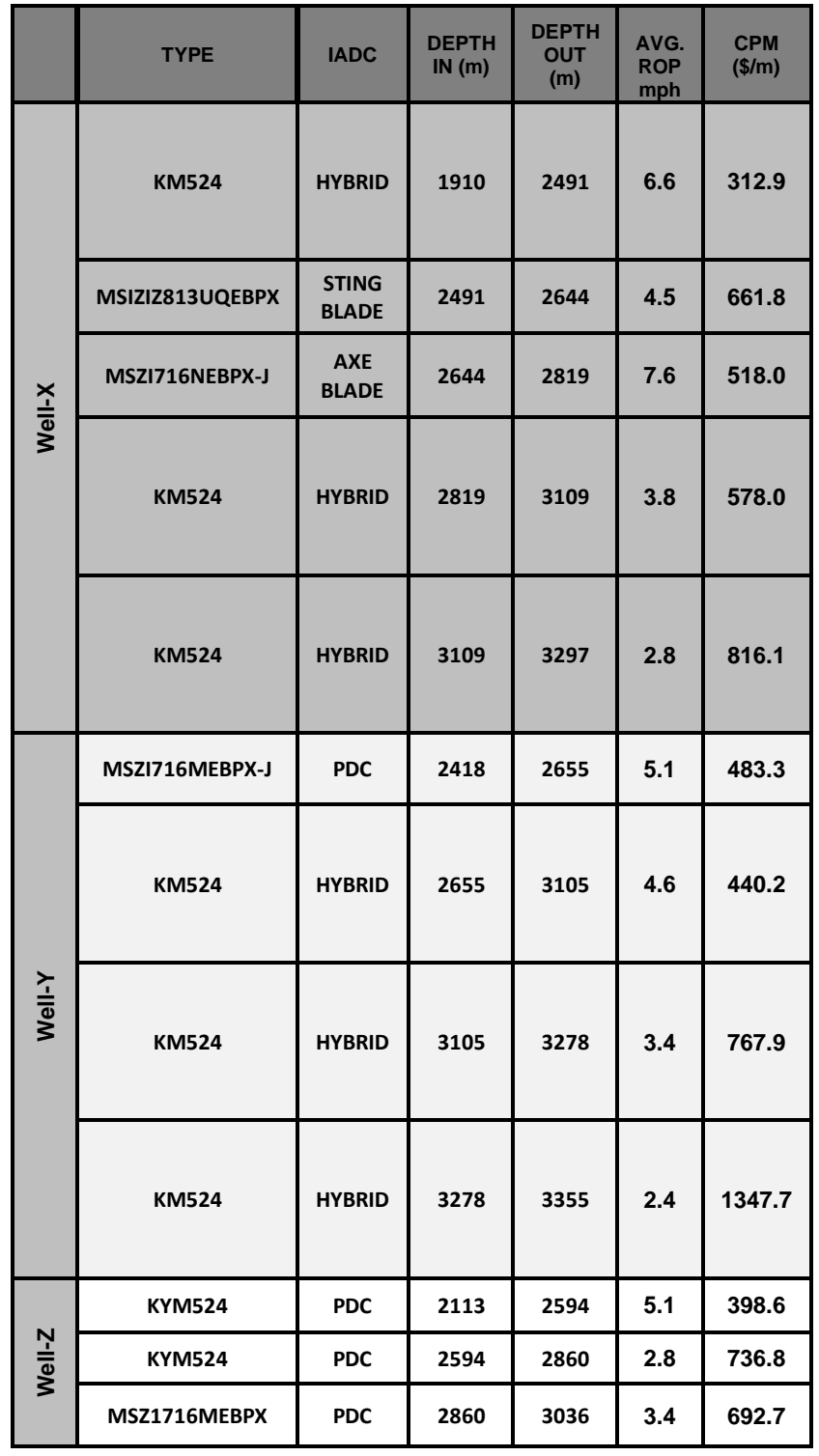

\section{ROP Modelling}

Well-X is modelled by multiple regression technique. The computed correlation coefficients are provided in table 2. The extracted Coefficients from multiple regression ROP model are reliable. It's noticed that the b1 (WOB), b2 (RPM) \& b4 (JIF) are of positive values, on the other hand, b3 (TRQ) and b5 (UCS) are of negative values which is reliable. By implementing 
the multiple regression coefficients provided from multiple regression workflow applied on the original well (well-X), a new ROP profile could be predicted and when the new computed ROP profile is compared with the actual ROP profile, the result shows a good match, as shown in Figure (4). Wells ( $Y \& Z$ ) have the same drilling conditions (drill bits, bottom hole assemblies and geological structure) of the reference well (well-X), so the coefficients extracted from well-X ROP model could be applied on these wells to test its reliability and accuracy. The results show that the well-X based model when applied on well- $Y$ predicts excellent match. The predicted ROP appears to match obviously with the actual ROP, although it deviates in some sections. In addition to the clear match between the predicted and actual ROP, the average predicted and actual ROP values using the model are close to each other's (average predicted ROP is $5.03 \mathrm{mph}$ and the average actual ROP is $5.01 \mathrm{mph}$ ), shown in Figure (5). In well-Z, the predicted ROP profile appears to correlate very well with the actual one. The average actual ROP is $5.47 \mathrm{mph}$ and the average predicted ROP is $5.57 \mathrm{mph}$, shown in Figure (6).

Table 2 Calculated regression coefficients based on reference well $(X)$

\begin{tabular}{|l|l|}
\hline Coefficient & Coefficient Value \\
\hline Intercept, b0 & 12.14776608 \\
\hline b1 (WOC) & 0.089151953 \\
\hline b2 (RPM) & 0.066000126 \\
\hline b3 (TRQ) & -0.081773106 \\
\hline b4 (JIF) & 0.000870105 \\
\hline b5 (UCS) & -0.000688606 \\
\hline
\end{tabular}

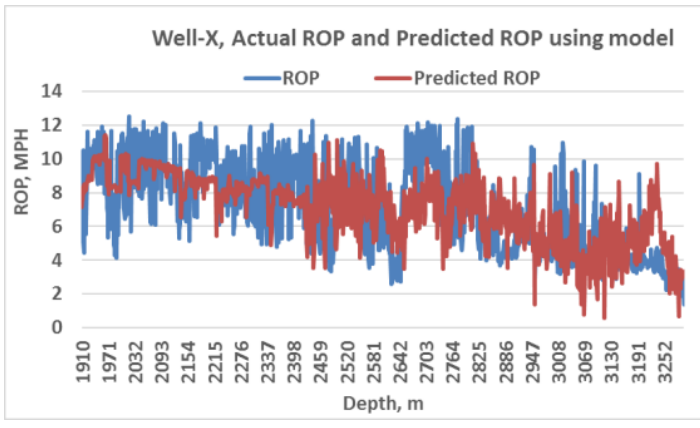

Figure 4 multiple regression method applied on well-X

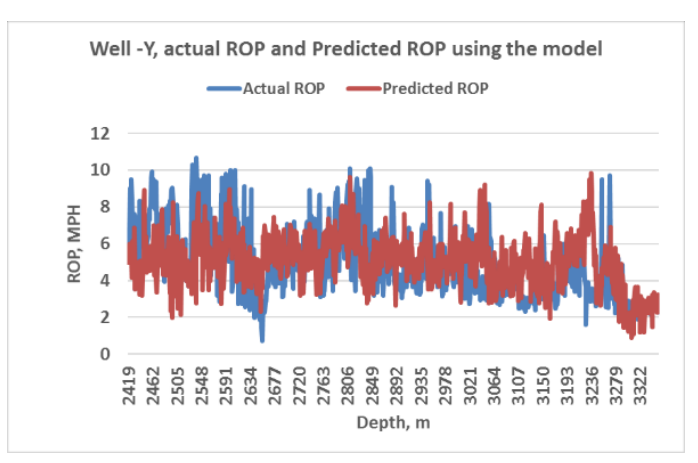

Figure 5 multiple regression method applied on well-Y using coefficients from well-X

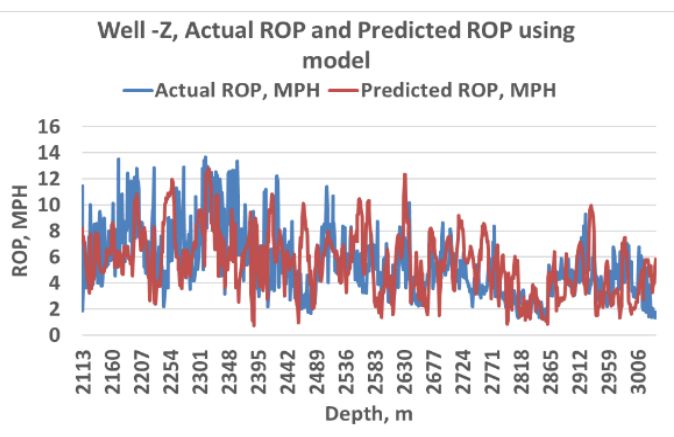

Figure 6 multiple regression method applied on well-Z using coefficients from well-X

\section{Optimization Drilling Parameters}

Determining the most effective drilling parameters on the drilling performance is easily carried out by the developed ROP model. As a part of

modelling, predicting which drilling parameters have a noticeable effect on the penetration rate is the upmost target of any ROP model.

The controllable drilling parameters that have been used in the ROP model (i.e. WOB, RPM \& JIF) are increased by $10 \%$ one by one, and then the ROP model has been carried out on the new drilling parameters one by one. The new predicted ROP values after increasing the drilling parameters are compared with the original predicted ROP values. The results of increasing the drilling parameters are plotted against the modelled ROP, as shown in Figure (7) which shows the effect of increasing the drilling parameters on ROP values for wells (X, Y\& Z).

By finding out the drilling parameters that have the highest effect on the penetration rate, the new averages ROP are calculated, and the saved time and cost could be defined well. From Figure (7), we can conclude that the RPM has the highest impact on the penetration rate then the $\mathrm{WOB}$ and the least one is the JIF.

Figure 8 shows the variations in on bottom drilling time when the increase in drilling parameters is applied. The results are an increase in average ROP with $14 \%$ and a reduction in drilling time with $14 \%$ when the drilling parameters (WOB, RPM \& JIF) are increased with $10 \%$.

Figure 9 shows that the saved cost when the drilling parameters (WOB, RPM \& JIF) are increased by $10 \%$ varies from 30 to 48 thousand USD (32-52 \$/m) which represents about $10-14 \%$ of the total drilling cost (the cost has been calculated based on assumption that the average rig rate per hour is $1,800 \$)$. 


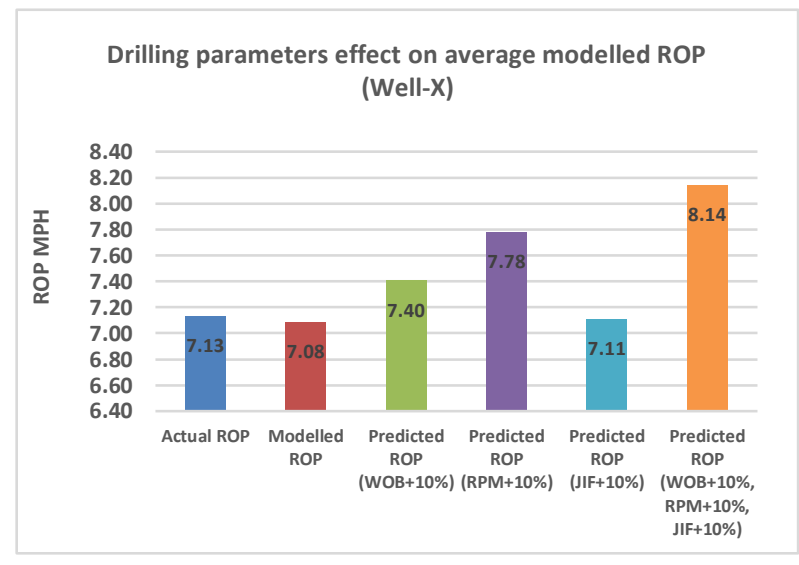

Figure 7 Average ROP values showing the effect of changing drilling parameters (well-X)

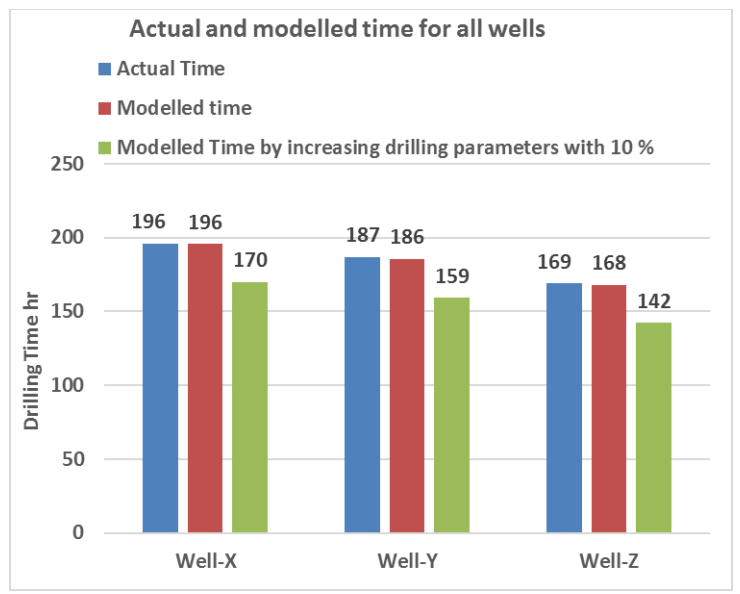

Figure 8 Actual and modelled time for the three wells

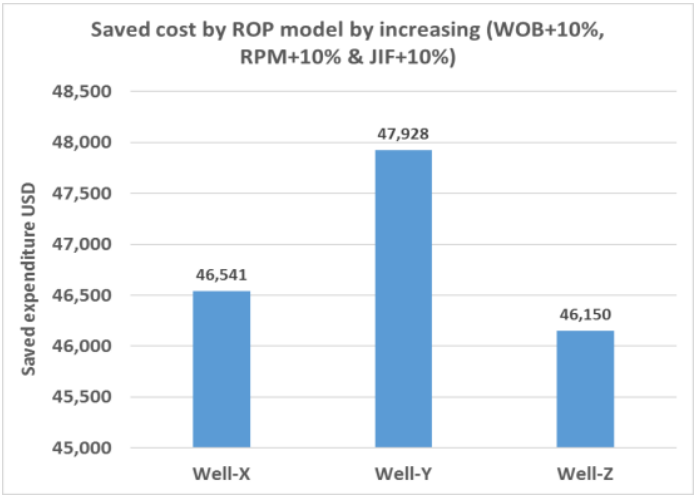

Figure 9 Saved cost when the drilling parameters (WOB, RPM \& JIF) are increased by $10 \%$.

\section{Conclusion}

The analysis showed that hybrid bit (kymera: KM524) had achieved high performance in drilling 8.5" hole in Sinai Oil Field. The kymera drill bits offered higher overall ROP $(2.5 \mathrm{mph}$ in the very hard formation and $6.6 \mathrm{mph}$ in medium hard formation), lower vibration, better directional control, longer drilled interval $(200 \mathrm{~m}-580 \mathrm{~m})$ and low average cost per drilled meter ( $350-700 \mathrm{~S} / \mathrm{m}$ ).

ROP model has been developed, to predict the ROP values before drilling, to be used in optimization of drill bits' performance in the forthcoming drilled wells. When the model has been checked and tested on two nearby wells, it has shown high level of accuracy and reliability. The ROP model has been used to optimize the drilling parameters as it relates the ROP value with increasing the drilling parameters. The developed ROP model has used to predict the effectiveness of each drilling parameter and its impact on the ROP values. The ROP model showed that when the drilling parameters (WOB, RPM \& JIF) are increased with $10 \%$, the saved time would be $18-27$ hour per and the saved cost would be $30,000-48,000$ $\$(32-52 \$ / \mathrm{m})$.

\section{Funding sources}

This research received no external funding.

\section{Conflicts of interest}

There are no conflicts to declare.

\section{References}

[1] D. T. Nguyen, (2012), Drill Bits Technology, Introduction of the new Kymera Hybrid Bit (chapter 1, P.5), University of Stavanger: Faculty of Science and technology.

[2] B. H. Ahmed Ismail and Waleed Mostafa, (2014) "New Hybrid Drill Bit with Innovative technology improves drilling efficiency in challenging Jordanian Project, SPE-172169-MS.

[3] P. Boryczko, (2012), Drill bit selection and optimization in exploration well 6507/6-4A in the Nordland Ridge Area.," University of Stavanger.

[4] H. W. university, (2005), Drilling engineering-heriotwatt university, (Chapter 5, P. 30)

[5] Dale E. Berger (2003), Introduction to Multiple Regression", (Chapter 1, P.8), Claremont Graduate University.

[6] H. Rabia, (2002), Well Engineering and Construction (chapter 10, P.326).

[7] "E. Momeni, D. J. Armaghani, M. Hajihassani, and M. F. M. Amin, (2015), Prediction of uniaxial compressive strength of rock samples using hybrid particle swarm optimization-based artificial neural networks measurement, (vol. 60, pp. 50-63). 